\title{
Depth Dependence of the Spatial Resolution in Scanning Transmission Electron Microscopy Experiments
}

\author{
Andreas Verch ${ }^{1}$ and Niels de Jonge ${ }^{1,2}$. \\ 1. INM - Leibniz Institute for New Materials, Campus D2 2, 66123 Saarbrücken, Germany \\ 2. Department of Physics, University of Saarland, Campus A5 1, 66123 Saarbrücken, Germany
}

Scanning transmission electron microscopy (STEM) is mostly used for very thin (few tens of nanometers or less) specimens. However, for a range of scientific applications, it is important to image through thicker samples. For example, STEM of specimens in liquid involves thicknesses of a few hundreds of nanometers to several micrometers. Beam broadening occurs when the thickness is larger than the mean free path length for elastic scattering in the materials under investigation. As a result, an electron beam not focused to the top layer (with respect to a downward traveling electron beam) becomes substantially broadened thus reducing the spatial resolution. But also the spatial resolution for objects in the top layer is influenced by the material beneath as the contrast and thus the signal-to-noiseratio decreases with increasing sample thicknesses. Both effects reduce the spatial resolution at a given electron dose in a STEM experiment. The resolution for imaging an object either at the top or at the bottom of thick specimen was determined experimentally, via simulations, and in various analytical models [1]. An important question is what happens for particles at a certain vertical position within a thick specimen. The effect of beam broadening on the resolution in this case was approximated by a preliminary analytical model [2] but not yet verified experimentally. Here, we show beam-broadening data obtained for nanoparticles at certain vertical distances within a thick sample.

As experimental model system we chose to study gold nanoparticles embedded in a solid aluminum film. The advantage of this approach is that the gold nanoparticles are immobilized at specified vertical positions in an electrically conducting matrix. Multiple layers of aluminum were deposited by physical vapor deposition (PVD) on silicon chips featuring thin $(50 \mathrm{~nm})$, electron beam transparent silicon nitride windows in the center through which the imaging was done. These silicon chips are identical to those also used in liquid STEM experiments. Gold nanoparticles of 5-10 $\mathrm{nm}$ in diameter were placed between the individual layers of aluminum. In addition, gold nanoparticles and nanorods were deposited on top of the aluminum film as well as at the bottom, enabling us to determine the thickness of the aluminum film via tilting of the specimen holder. The setup of our experiments is illustrated in Figure 1. The experiments were performed using a $\mathrm{C}_{\mathrm{S}}$-corrected STEM/TEM (ARM200f, JEOL. Japan) at $200 \mathrm{kV}$ acceleration voltage.

Our experiments indeed showed that the image resolution is strongly depended on the vertical position of the gold nanoparticles within the aluminum matrix. The deeper the particles within the Al support were the lower the image resolution became. This is illustrated by the electron micrographs shown in Figure 2, where the same particles are shown from both sides of the silicon chip. In a) the particles were below $0.48 \mu \mathrm{m}$ of $\mathrm{Al}$, while there was only $0.12 \mu \mathrm{m}$ of $\mathrm{Al}$ above the nanoparticles in $\mathrm{b}$ ). The total thickness of the supporting material was $0.6 \mu \mathrm{m}$ in both cases. The intensity profiles over the particle for both cases are shown in c). Interestingly, the change in the image resolution was not reflected in the full widths at half maximum of the intensity profiles, which were almost the same for particles close to the top surface and those deeper in the aluminum matrix. However, the distance from $25 \%$ to $75 \%$ of the maximum intensity $\left(\mathrm{d}_{25-75}\right)$ in the intensity profiles significantly changed with the vertical depth of the 
particles. This finding is in good agreement with the model by Schuh et al.[2]. [3]

References:

[1] H Demers et al., Microsc Microanal 18 (2012), p. 582.

[2] T Schuh and N de Jonge, C R Phys 15 (2014), p. 214.

[3] We thank E. Arzt for his support through INM. Research in part supported by the Leibniz Competition 2014.
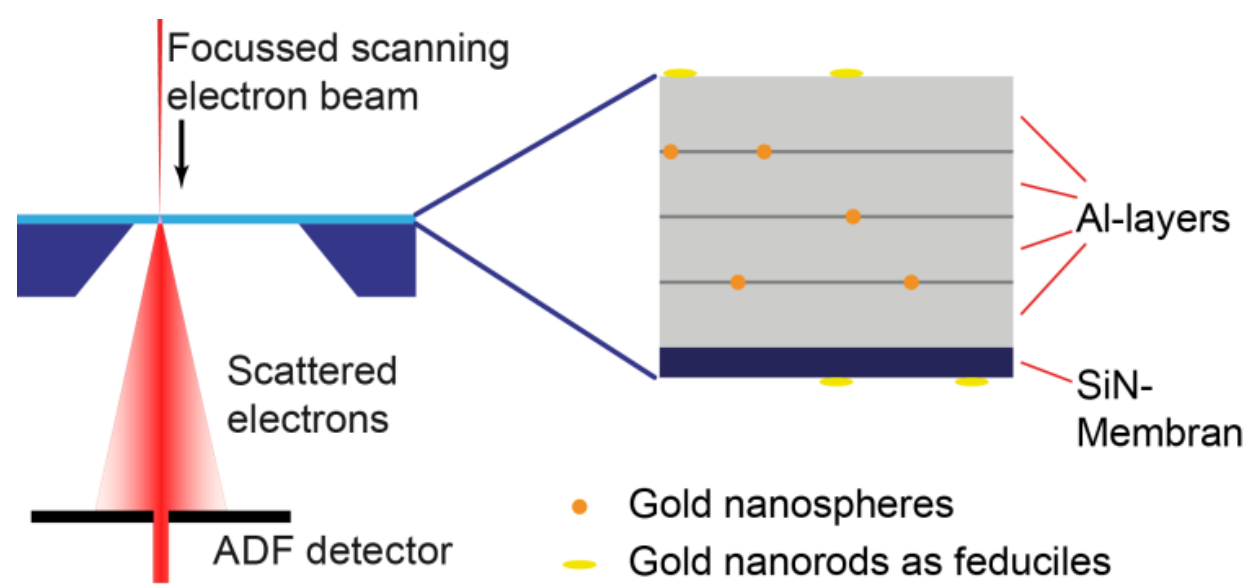

- Gold nanospheres

- Gold nanorods as feduciles

Figure 1: Schematic drawing of sample studied using (STEM) with annular dark field (ADF) detection. A Si microchip with an electron transparent $\mathrm{SiN}$ window was coated with multiple layers of $\mathrm{Al}$ by means of physical vapor deposition (PVD). Gold nanoparticles of sizes between 5 and $10 \mathrm{~nm}$ were deposited between the different layers of Al.
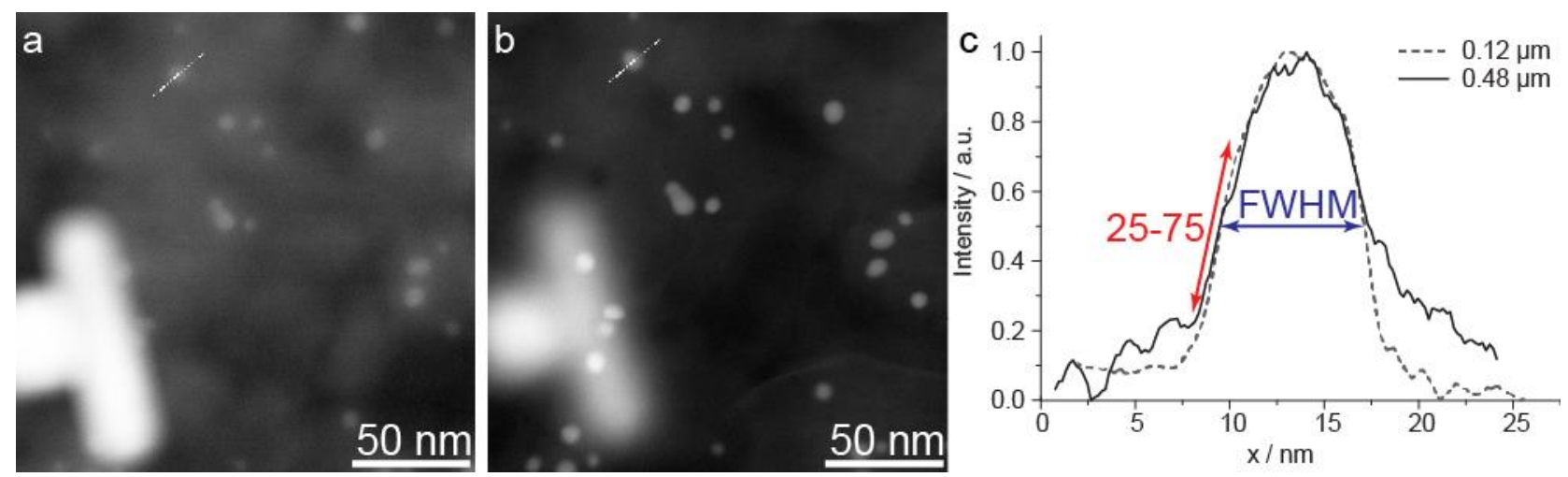

Figure 2: STEM of nanoparticles embedded in Al matrix. a, b) Micrographs of the same region but acquired from opposite beam directions with respect to the $\mathrm{SiN}$ window (by flipping microchip). The vertical depths of the particle indicated with the dashed line were $0.48 \mu \mathrm{m}$ in a), and and $0.12 \mu \mathrm{m}$ in b), respectively. The total thickness of the supporting material was $0.6 \mu \mathrm{m}$. c) Normalized line profiles along the dashed lines in a) and b) and the definitions for the full width at half maximum (FWHM, blue) and the $\mathrm{d}_{25-75}$ (red). 